Monatsschrift f. Geburtshülfe u. Gynäkologie 1934;97:I-IV

\title{
Contents, Vol. 97, 1934
}

Inhaltsverzeichnis.

Or¹/8ínalarbeiten. $\quad$ S $\theta$ it $\theta$

Beaufays, J., Über habituelles Hydramnion 221

Borissowa, N. A., Die Kesultate der Behandlung von Kolpitiden und

Erosionen bei Schwangeren mit Biolaktin 159

v. Bud, Georg, Fokale Erkrankungen bei Schwangerschaftstoxikosen 285 Burger, K., Der

Einfluß des Geburtsvorganges und der geburtshilfliehen

Operationen auf das Neugeborene 75

Conill, V., Über die Tubeninnervation 266

Czygak, Josef, Zur Histopathogenese der Teerzysten, zugleich ein

Beitrag zur Pathologie der Bartholinischen Druse $\quad \cdots 274$

Eufinger, H., und B. Schwemmler, Emulsionsbereitschaft des Serums

beim Genitalkarzinom der Frau 335

F r e s s e 1, J., Die Bedeutung der durch Schwangerschaft und Geburt

bedingten Kreislaufveränderung für die Entstehung der Eklampsie 14 Fuge, Kurt,

Untersuchungen über die Magenazidität bei Frauen mit

einem Genitalkarzinom 37

Gigowsky, E. E., Zur Histopathologie der sogenannten sklerozystischen

Ovarien 226

Hajek, 0., und K. Wepsehek, Untersuchungen über den Einfluß von

langdauernden Hypophysenvorderlappenhormongaben in Form von

Prolan auf lebenswichtige Organe im Meerschweinchenversuch . . . 217 Helm, Eberhard, Über

die geburtshilfliche Landesstatistik des Staates

Hamburg in den Jahren 1901-1930 129

Holzapfel, Karl, Seltene Gynatresie 89

-, Vulvaverschluß duvch Verbrennung

-, Zur Behandlung der klimakterischen Blutungen mit Vaporisation . . 269

Horn, Luise-Lotte, Schlofi $1 / 1 / 8$ rscher Tumor 71

Naumes, E., siehe Spiegler, Budolf.

$\mathrm{Ne}$ be sky, 0., Unsere Erfahrungen bei derStrahlentherapie des Carcinoma

colli uteri 317

Penkow, G. W, siehe Tschertok, B. A.

Poljanskaja, A. N., Zur Frage über die rationelle Behandlung von

Mastitiden und Fisteln der Brustdrüse mit Diathermie $\quad 44$

Purper, Gerhard Friedrich, Das Weltmannsche Koagulationsband

bei Adnexentzündungen 138

Bhemann, Franz, Über die Berechtigung der prophylaktischen, pla-

stischen Operationen bei Doppelbildung der Gebärmutter 1

Schepetinsky, Anna, Über K- und Ca-Gehalt des Blutes im Laufe 
des Menstruationszyklus . 83

Scheyer, H. E., Zur Kasuistik der kongenitalen Haut- und Knochen-

defekte am Schädeldach $\quad 65$

Schmidt, W. Th., Ein Beitrag zur intrauterinen Frucht-Schädigung . . 48 -, Über einen

erfolgreichen Fall von Ileumplastik bei Scheidenatresie 50 Schwemmler, B., siehe Eufinger, H.

Seitz, Ludwig, Der essentielle Schwangerschaftshochdruck als selb-

ständiges Krankheitsbild 325

Simonsitz,Géza, Über zwei Fälle schwerster puerperaler Gasbazillen-

allgemeininfektion 7

de Snoo, K., Die Nierenfunktion bei Schwangerschaftstoxikosen und

Eklampsie 253

Sommer, Stephan, Über die intrakutane Lebendvakzine-Behandlung

der weiblichen Gonorrhoe 339

Spiegler, Eudolf, und E. Naumes, Ein Beitrag zum Magnesium-

gehalt des Serums. (Unter Berücksichtigung einer neuen Methode.) 22 Stähler, Fritz, Moderne

Schwangerenfürsorge im Kampf gegen den

Geburtenrückgang 189

Tietze, H., Fast ausgetragene interstitielle Gravidität 153

JY Inhaltsverzeichnis.

Seit $\theta$

Traube, Karl, Über Bestrebungen zur Herabsetzung der geburts-

hilflichen mütterlichen und kindlichen Mortalität 208

Tschertok, ß. A., und G. W. Fenkow, Die Wirkung der Hormone

des Hypophysenvorderlappens auf nicht funktionierende Eierstöcke

des Weibes 146

Umnova, W. P., Ein Fall von Selbstheilung zweier extrauteriner

Schwangerschaften mit Ausgang beider in elf und neun Jahre alte

Lithopaedien 349

Welsch, Kurt, Über den G1/8burtsverlauf nach Beckenfrakturen . . . 197

Wepschek, K., siehe Hajek, 0.

Grenzgebíete der Geburtshilfe und Gynäkologîe.

Der Stoffwechsel bei Schwangeren (IV). Jahresübersicht. Von Privatdozent

Dr. A. Bock, Berlin 53

Die histologischen Forschungen über die weiblichen Genitalorga $\pi$. Literatur-

übersicht über das Jahr 1933. Von Hans Otto Neumann, Mar

burg (Lahn) 94

Physikalische Heilmethoden, einschließlich der Hydrotherapie (IV). Von

Hermann Franken, Freiburg i. Br 106

Lebererkranku $\pi$ gen, uropoetisch.es System and Nierenerkrankungen in ihren

Beziehungen zur Geburtshilfe und Gyлäkologie. Von Privatdozent

Dr. Hans Rupp, Bonn 166

Kreislaufforschung des vergangenen Jahres. Von Hans Bait zer, Tubingen 231

Konstitutionstherapie unter besonderer Berücksichtigung der Leibesübungen.

Von Fritz Stähler, Frankfurt a. M 293 
Tuberkulose als Grenzgebíet der Geburtshilfe und Gynäkologîe (IV). Von Prof. Dr. K. H a n s e n , Lübeck, und Prof. Dr. F. Schultze-E, honhof, Heidelberg 354

Soziale Gynäkologîe und Geburtshilfe (IV). Von Fr. Chr. Geller, Breslau 363

Sítzungsberichte

aus geburtshilflich-gynäkologischen Gesellschaften.

58. Tagung der Oeutschen Gesellschaft für Chirurgie, Berlin. 4.-7. April 1934.

Referent: Dr. L. Waldeyer, Berlin

113

Bericht fiber die 25. Tagung der Deutschen R $\delta$ ntgengesellschaft in Baden-

Baden. 14.-15. April 1934. Von Dr. Fritz Stähler, Frankfurt a. M. 238

46. Kongreß der Deutschen Gesellschaft für innere Medizin in Wiesbaden.

9.-12. Mai 1934

302

Gesellschaft für Geburtshilfe und Gynäkologîe zu Berlin. Sitzungen vom

12. Januar und 16. März 1934 308

- , Sitzung vom 10. November 1933

-, Sitzung vom 8. Dezember $1933 \quad 178$

-, Sitzung vom 16. Februar $1934 \quad 244$

Gynäkologische Gesellschaft zu Breslau. Sitzung vom 23. Januar 1934 . . 116

-, Sitzung vom 27. Februar 1934

242

-, Sitzung vom 15. Mai 1934

372

Geburtshilflich-Gynäkologische Gesellschaft in Wien. Sitzung vom 27. Fe bruar 1934 119

-, Sitzung vom 13. März 1934

176

-, Sitzung vom 15. Mai 1934

249

Buchbesprechungen

Personalien und Tagesnachrichten

$64,127,186,250,315,87464,127,188,252,316,376$ 